\title{
2000-2017年新疆天山植被水分利用效率时空特征 及其与气候因子关系分析
}

\author{
艾则孜提约麦尔・麦麦提 ${ }^{1}$ 玉素甫江・如素力 ${ }^{1,2^{*}}$ 何 辉 ${ }^{1}$ 拜合提尼沙・阿不都 \\ 克日 木 $^{1}$
}

${ }^{1}$ 新疆师范大学地理科学与旅游学院流域信息集成与生态安全实验室, 乌鲁木齐 $830054 ;{ }^{2}$ 新疆干早区湖泊环境与资源重点实验室, 乌鲁木齐 830054

摘 要 水分利用效率(WUE) 是衡量生态系统碳水循环耦合程度的重要指标, 估算新疆天山及南北主要绿洲的植被WUE并 分析其时空变化规律, 探索其影响因素, 对该区域生态系统保护、农业水资源的合理利用与开发等方面具有重要的意义。基 于MODIS遥感数据、气象数据和土地利用类型数据, 分析新疆天山近 18 年植被WUE时空变化特征以及与气候因子的关系。 结果表明: (1) 2000-2017年新疆天山植被WUE变化范围为 $0.84-1.34 \mathrm{~g} \cdot \mathrm{mm}^{-1} \cdot \mathrm{m}^{-2}$, 多年均值为 $1.11 \mathrm{~g} \cdot \mathrm{mm}^{-1} \cdot \mathrm{m}^{-2}$, 整体呈减少趋 势, 变化率为 $-0.0141 \mathrm{~g} \cdot \mathrm{mm}^{-1} \cdot \mathrm{m}^{-2} \cdot \mathrm{a}^{-1}$; 空间分布具有较强的垂直地带性规律, $1000 \mathrm{~m}$ 以上的区域随着海拔的升高而减少。(2) 植被WUE年内变化呈单峰型变化格局, 具有明显的季节性差异, 表现为: 夏季>春季>秋季>冬季。(3)相关分析和统计结果表 明, 新疆天山植被WUE时空变化受到气温影响的区域占 $33.23 \%$, 受降水影响的区域占 $8.57 \%$, 受气温和降水综合强影响的区 域占 $5.63 \%$, 气温和降水综合弱影响的区域占 $13.13 \%$; 因此气候因素中气温在新疆天山植被WUE的变化中起到主导作用。(4) 水田与旱地水分利用效率随着时间变化呈持续减少趋势, 并且这些区域基本上受到非气候因子的影响, 说明当地人类活动存 在不合理性。

关键词 水分利用效率; 植被; 时空分布; 气候因子; 新疆天山

艾则孜提约麦尔・麦麦提, 玉素甫江·如素力, 何辉, 拜合提尼沙·阿不都克日木 (2019). 2000-2017年新疆天山植被水分利用效率时空特征及其与气候因 子关系分析. 植物生态学报, 43, 490-500. DOI: 10.17521/cjpe.2019.0006

\section{Spatio-temporal characteristics of vegetation water use efficiency and its relationship with climate factors in Tianshan Mountains in Xinjiang from 2000 to 2017}

Aizezitiyuemaier MAIMAITI ${ }^{1}$, Yusufujiang RUSULI ${ }^{1,2^{*}}$, HE Hui $^{1}$, and Baihetinisha ABUDUKERIMU ${ }^{1}$

${ }^{1}$ Laboratory of Information Integration and Ecological Safety, Institute of Geographical Science and Tourism, Xinjiang Normal University, Ürümqi 830054, China; and ${ }^{2}$ Xinjiang Key Laboratory of Lake Environment and Resources in Arid Zone, Ürümqi 830054, China

\section{Abstract}

Aims Water use efficiency (WUE) is a key index to measure the coupling degree of carbon and water cycle in ecosystems. The WUE of vegetation in Tianshan Mountains in Xinjiang and the north and south sites of the main oasis was estimated and then the spatio-temporal distribution of vegetation WUE was analyzed to explore its influencing factors, which will be of great significance to the protection of ecosystem and the rational utilization and development of agricultural water resources in this region.

Methods This study used data from moderate-resolution imaging spectroradiometer (MODIS), meteorological and land use type data to estimate the vegetation WUE. The spatio-temporal characteristics of vegetation WUE were analyzed in Tianshan Mountains in Xinjiang over the last 18 years, and the relationship of WUE with climatic factors was evaluated.

Important findings The results indicated that: (1) From 2000 to 2017, the average annual vegetation WUE for Tianshan Mountains in Xinjiang was $1.11 \mathrm{~g} \cdot \mathrm{mm}^{-1} \cdot \mathrm{m}^{-2}$, ranging from 0.84 to $1.34 \mathrm{~g} \cdot \mathrm{mm}^{-1} \cdot \mathrm{m}^{-2}$. As a whole, the annual decrease trend of vegetation WUE was $0.0141 \mathrm{~g} \cdot \mathrm{mm}^{-1} \cdot \mathrm{m}^{-2} \cdot \mathrm{a}^{-1}$, and vegetation WUE showed a strong vertical zonality in Tianshan Mountains in Xinjiang, as indicated by the decrease with the altitude above $1000 \mathrm{~m}$. (2) The vegetation WUE in Tianshan Mountains in Xinjiang showed a unimodal change pattern with significant seasonal difference, in order of summer $>$ spring $>$ autumn $>$ winter. (3) Correlation analysis and statistical results 
indicated that the dynamic change of vegetation WUE in Tianshan Mountains in Xinjiang was closely related to temperature and rainfall. The regions with vegetation WUE changes resulting from non-climate factors accounted for 39.26\% in Tianshan Mountains in Xinjiang. However, the factors of temperature and precipitation contributed to the change of vegetation WUE as $33.23 \%$ and $8.57 \%$, respectively. On the other hand, the combination of temperature and precipitation with heavy impact and light impact contributed to WUE by $5.63 \%$ and $13.13 \%$, respectively. Overall, temperature played the most important role among all climate factors in the changes in vegetation WUE. (4) The WUE of paddy field and dryland decreased continuously with time, and these areas were mainly affected by non-climatic factors, suggesting the irrationality in local human activities.

Key words water use efficiency; vegetation; temporal and spatial distribution; climate factor; Tianshan Mountains in Xinjiang

Maimaiti A, Rusuli Y, He H, Abudukerimu B (2019). Spatio-temporal characteristics of vegetation water use efficiency and its relationship with climate factors in Tianshan Mountains in Xinjiang from 2000 to 2017. Chinese Journal of Plant Ecology, 43, 490-500. DOI: $10.17521 /$ cjpe.2019.0006

水分利用效率(WUE) 是指植物消耗单位质量的 水分所固定 $\mathrm{CO}_{2}$ 或生产干物质的量(胡中民等, 2009), 它是陆地生态系统对气候变化响应敏感的重要指标 (王庆伟等, 2010), WUE耦合了陆地生态系统碳循环 和水循环两个关键生态系统过程(Gang et al., 2016), 不仅可以反映二者之间的相互关系, 而且还能够解 释陆地生态系统对全球气候变化的响应(杜晓铮等, 2018)。因此, 研究WUE的动态变化及其对气候因子 的响应, 对于评估区域碳水耦合关系及全球气候变 化对生态系统的影响具有重要意义(张远东等, 2016)。

由于学者们对生态系统WUE的内涵理解不同 以及数据获取手段的差异, 估算WUE的方法有所不 同(王芳等, 2018)。在生态系统或区域尺度, 常采用 总初级生产力 $(G P P)$ 或净初级生产力 $(N P P)$ 或净生 态系统交换 $(N E E)$ 与蒸散发 $(E T)$ 的比值来进行估算。 其中, 采用 $G P P$ 与 $E T$ 的比值计算 $W U E$ 是最为经典 的计算方式(Jassal et al., 2009; 仇宽彪和成军锋, 2015; 张远东等, 2016), 该定义通常用在月、年等长 时间尺度上分析生态系统的碳水耦合特征及其对环 境变化的响应(刘晓等, 2017)。因此, 本研究采用 $G P P$ 与ET的比值估算植被WUE。

WUE不仅受系统内部植被的调控, 还与外界环 境条件密切相关, 同时又受到两者相互作用的影 响。在外界环境条件中, 气温、降水、太阳辐射等 均是影响植被WUE的关键气候变化因子(李辉东等, 2015; 安佑志等, 2016; 李明旭等, 2016; 李肖娟等, 2017)。在全球气候变化背景下, 极端气候事件的频 繁出现对我们研究生态系统WUE提出了新的要求 (陈洪滨和了丽军, 2005; 陈新光等, 2007; 王冀等, 2012; 郑景云等, 2014)。因此, 生态系统WUE的时
空变化及其对气候变化响应的研究受到国内外诸多 学者的广泛关注(卢玲等, 2007; Klein et al., 2013; 李辉东等, 2015; 位贺杰等, 2016)。

新疆天山地形地貌十分复杂, 山区和南北两侧 的气候、土壤条件以及植被覆盖率等方面具有非常 明显的差异性, 作为对气候变化高度敏感的干旱区 植被系统, 天山地区的植被生态不仅影响着区域生 态环境和畜牧业水平, 更对整个中国西北和中亚地 区的生态稳定及经济发展起到重要作用。目前针对 该区域的植被生态展开的研究多是关于植被覆盖 度、碳储量、生物量(吴晓全等, 2016; 玛地尼亚提・ 地里夏提等, 2018; 李艳菊等, 2019)等方面的, 针对 植被WUE的研究尚未见到。因此, 本文以新疆天山 为研究区, 利用MOD16 ET和MOD17 GPP数据估算 该区域的植被WUE, 探索该区域植被WUE时空变 化特征及其与气候因子的关系, 研究结果有助于对 区域植被水分利用效率时空变化的理解, 并对该区 域生态系统保护、农业水资源的合理利用与开发等 方面具有重要的指导意义。

\section{1 研究区概况}

新疆天山位于新疆中部, 主体呈东西走向, 山 间有盆地, 如哈密盆地、吐鲁番盆地, 西侧喇叭口地 形为伊犁河谷。天山作为南北疆气候的分界岭, 天 山以北属于温带, 以南属于暖温带, 南北在气温、降 水变化上存在较大的差异。因此本研究主要考虑天 山的地形地貌、南北植被覆盖度以及流域分布等情 况, 划分了适合本研究目标的研究区空间范围; 整 个研究区地处 $39.85^{\circ}-47.28^{\circ} \mathrm{N}, 75.32^{\circ}-97.02^{\circ} \mathrm{E}$, 总面积约631280 km², 海拔在-170-6998 $\mathrm{m}$ 之间, 年平均气温在 $5.6{ }^{\circ} \mathrm{C}$ 左右; 年降水量 37.2-912.5 
$\mathrm{mm}$ 。天山北部包括艾比湖、额敏河以及古尔班通 古特沙漠, 南部包括阿克苏河、渭干河、塔里木河 中游和开孔河等流域; 研究区内还有我国最大的内 陆淡水湖博斯腾湖、新疆最大的咸水湖艾比湖和新 疆海拔最高的高山湖泊赛里木湖; 总体上, 天山是 南北平原区的“湿岛” (魏文寿和胡汝瀷，1990), 是 天山南北许多内流河流的补给来源, 滋养着周边 的绿洲生态系统, 影响着局地的生态和社会经济 环境。

\section{2 数据来源和研究方法}

\section{1 数据来源}

\subsubsection{MODIS数据}

MOD16A2和MOD17A2H产品数据均来自于美 国陆地过程分布式数据档案中心(LPDAAC), 空间 分辨率均为 $500 \mathrm{~m}$, 时间分辨率为 $8 \mathrm{~d}$, 遥感卫星轨 道号为 $\mathrm{h} 24 \mathrm{v} 04$ 和 $\mathrm{h} 23 \mathrm{v} 04$, 时间跨度为 2000 年 2 月 28 日到2017年12月31日。利用MRT (MODIS Reprojection Tools)将下载的MOD16A2和MOD17A2H数 据进行格式转换、重投影和影像镶嵌等预处理。该 数据相对于MODIS C5数据, 不仅空间分辨率提高 到500 m, 还在一定程度上消除了因卫星传感器老 化而造成的数据衰减和失真问题(Lyapustin et al., 2014)。这两种产品数据均包含各自的数据质量控制 文件(ET_QC_500 m和Psn_QC_500 m), 其表示不同 区域ET和 GPP产品的质量可靠性(Heinsch et al., 2003; Running \& Zhao, 2015; Running et al., 2018), Wang等(2017)针对MODIS GPP产品数据在全球和 中国的精度进行了验证并证明该数据在中国区域有 一定的可靠性(Zhu et al., 2016; Wang et al., 2017 )。 阿迪来·乌甫等(2017)对MODIS ET产品在新疆区的 精度进行验证并证明了该数据的可靠性。

\subsection{2 气象数据}

气象数据来源于中国科学院资源环境科学数据 中心(http://www.resdc.cn)基于全国2 400多个气象 站点的日观测数据, 通过整理、计算和空间插值处 理生成了2000-2015年气温和降水量空间数据集。本 研究区共有 71 个气象站点, 空间分辨率为 $1 \mathrm{~km}$ 。考 虑到常用的插值方法由于地形起伏等因素使得数据 精度较低, 本文的数据使用ANUSPLIN软件, 该数 据已被诸多研究者认可并使用; 2016-2017年采用 TRMM3B43 (Tropical Rainfall Measuring Mission satellite) 的降水数据和全球陆面数据同化系统 (Global Land Data Assimilation System, GLDAS)的 GLDAS_NOAH $0.25^{\circ}$ 月合成气温数据。TRMM降水 数据的格式为HDF格式, GLDAS_NOAH $0.25^{\circ}$ 气温 数据的格式为.nc格式, 使用之前对两套数据进行格 式转换、投影转换以及重采样等一致化处理, 最终 得到与MODIS产品数据分辨率和投影系统相同的 空间数据。

\subsection{3 地形数据}

数字高程模型(DEM)数据由美国国家航空航天 局(NASA)与日本经济产业省(METI)合作完成的先 进星载热发射和反射辐射仪全球数字高程模型 ASTER GDEM V2.0 (分辨率为 $30 \mathrm{~m}$ )数据下载获取。

\subsection{4 矢量数据}

矢量数据来源于中国科学院资源环境科学数据 中心 (http://www.resdc.cn) 的三级流域空间分布数 据。

\section{2 研究方法}

\subsubsection{WUE的计算}

本文用 $G P P\left(\mathrm{~g} \mathrm{C}^{-2} \mathrm{~m}^{-2}\right.$ 和 $E T(\mathrm{~mm})$ 之比计算 $W U E$ $\left(\mathrm{g} \mathrm{C} \cdot \mathrm{mm}^{-1} \cdot \mathrm{m}^{-2}\right)$ :

$$
W U E=\frac{G P P}{E T}
$$

\subsection{2 偏差分析}

采用偏差分析法对新疆天山各栅格单元 2000-2017年植被WUE的波动水平进行分析，计算 公式为:

$$
P_{i j}=W U E_{i j}^{t}-\overline{W U E_{i j}}
$$

式中, $P_{i j}$ 表示 $i$ 行 $j$ 列的植被 $W U E$ 偏差值, $W U E_{i j}^{i}$ 为 $t$ 年 $i$ 行 $j$ 列的植被 $W U E$ 值, $\overline{W U E_{i j}}$ 为 $i$ 行 $j$ 列的多年平 均植被WUE值, $t$ 为年份。

\subsection{3 趋势分析}

采用一元线性回归分析方法对2000-2017年新 疆天山植被WUE的动态进行变化趋势分析, 单个像 元多年回归方程中趋势线斜率即为年际变化率。计 算公式为:

$$
\text { Slope }=\frac{n \times \sum_{i=1}^{n}(i \times W U E)-\sum_{i=1}^{n} i \sum_{i=1}^{n} W U E_{i}}{n \times \sum_{i=1}^{n} i^{2}-\left(\sum_{i=1}^{n} i\right)^{2}}
$$




\subsection{4 相关性分析}

相关性分析是为了揭示要素间相互关系的密切 程度, 偏相关分析是在不考虑其他要素影响的前提 下计算两个变量的相关性。采用像元计算的偏相关 系数法分析了降水量和气温对植被WUE变化的影 响, 为了计算偏相关系数, 首先计算相关系数, 其 公式如下:

$$
R_{x y}=\frac{\sum_{i=1}^{n}\left(x_{i}-\bar{x}\right)\left(y_{i}-\bar{y}\right)}{\sqrt{\sum_{i=}^{n}\left(x_{i}-\bar{x}\right)^{2}} \sqrt{\sum_{i=}^{n}\left(y_{i}-\bar{y}\right)^{2}}}
$$

式中, $R_{x y}$ 是 $x, y$ 两个变量的相关系数; $x$ 和 $y$ 分别为第 $i$ 年的WUE和气候因子值; $\bar{x}$ 和 $\bar{y}$ 是两个变量的多年 平均值; $n$ 为年数。通过计算两变量的线性相关系数, 进而获得偏相关系数, 计算公式为:

$$
R_{x y, z}=\frac{R_{x y}-R_{x z} R_{y z}}{\sqrt{\left(x-R_{x z}^{2}\right) \sqrt{\left(x-R_{y z}^{2}\right)}}}
$$

式中, $R_{x y, z}$ 是将自变量 $z$ 固定后因变量 $x$ 与自变量 $y$ 的 偏相关系数。采用 $t$ 检验法对偏相关系数进行显著性 检验。

为了更进一步解析各要素之间的相互关系和 综合影响，采用复相关分析方法。其方法的计算公 式为:

$$
R_{x, y z}=\sqrt{1-\left(1-R_{x y}^{2}\right)\left(1-R_{x z, y}^{2}\right)}
$$

式中, $R_{x, y z}$ 是因变量 $x$ 和自变量 $y, z$ 的复相关系数; $R_{x y}$
表示 $x$ 与 $y$ 的线性相关系数, $R_{x z, y}$ 表示固定自变量 $y$ 后 因变量 $x$ 与自变量 $z$ 的偏相关系数。对此结果采用 $F$ 检验法进行显著性检验。

\section{3 结果和分析}

\section{1 新疆天山植被WUE年际时空分布特征} 从新疆天山2000-2017年GPP、ET及 $W U E$ 年际 变化(图 1)可知, $G P P$ 年均值波动范围为 451.29$576.31 \mathrm{~g} \mathrm{C} \cdot \mathrm{m}^{-2}$, 多年平均值为 $524.49 \mathrm{~g} \mathrm{C} \cdot \mathrm{m}^{-2}$, 整体 呈现增长趋势, 变化率为 $2.149 \mathrm{~g} \mathrm{C} \cdot \mathrm{m}^{-2} \cdot \mathrm{a}^{-1}$, 但并不 显著; $E T$ 为 $266.51-342.65 \mathrm{~mm}$, 多年平均值为 301.43 $\mathrm{mm}$, 整体呈现较明显的增长趋势 $(p<0.01)$, 变化 率为 $2.968 \mathrm{~mm} \cdot \mathrm{a}^{-1}$; 植被 WUE 的年均变化范围为 0.84-1.34 $\mathrm{g} \mathrm{C} \cdot \mathrm{mm}^{-1} \cdot \mathrm{m}^{-2}$, 多年均值为 $1.11 \mathrm{~g}$ $\mathrm{C} \cdot \mathrm{mm}^{-1} \cdot \mathrm{m}^{-2}$, 整体呈显著下降趋势 $(p<0.01)$, 变化 率为 $-0.0141 \mathrm{~g} \mathrm{C} \cdot \mathrm{mm}^{-1} \cdot \mathrm{m}^{-2} \cdot \mathrm{a}^{-1}$; 说明 $E T$ 的增长率比 $G P P$ 大是新疆天山植被 $W U E$ 年际变化下降的直接 因素。

利用偏差分析法分析2000-2017年各年植被 WUE偏离多年平均水平的程度(图2), WUE偏差值呈 现较复杂的波动状态，其中2003、2005、2010、2011、 2013年和最后3年的偏差偏离平均水平的程度较大, 2000-2002年、2005-2008年、2013和2014年的偏差 值均高于平均水平，其中2013年的植被WUE值最高, 为 $1.34 \mathrm{~g} \mathrm{C} \cdot \mathrm{mm}^{-1} \cdot \mathrm{m}^{-2}$, 高于多年平均的 $20.72 \%$, 2011 和 2016 年的植被WUE值较低，分别为 $0.86 \mathrm{~g}$

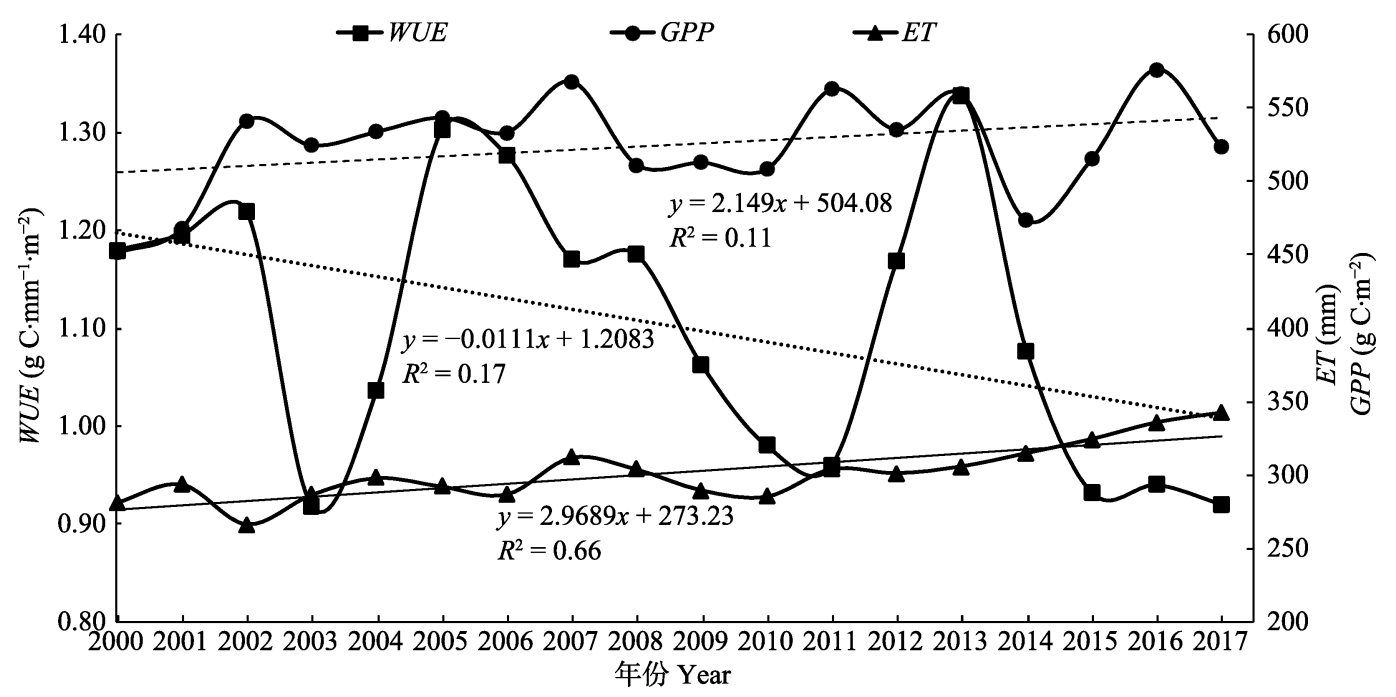

图1 2000-2017年新疆天山蒸发散(ET)、总初级生产力 $(G P P)$ 和水分利用效率(WUE)的年际变化。

Fig. 1 Annual variation of evaport transpration (ET), gross primary productivity $(G P P)$ and water use efficiency (WUE) in Tianshan Mountains Xinjiang during 2000 to 2017. 


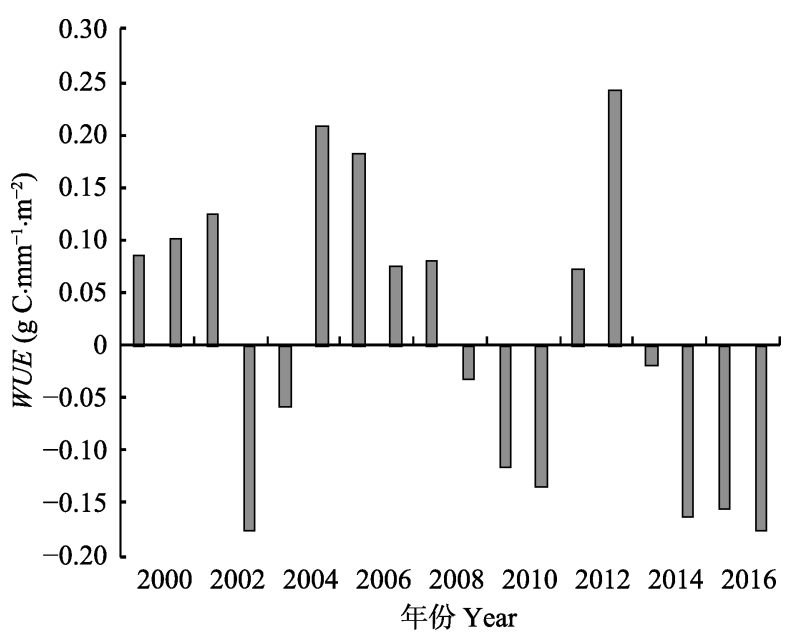

图2 新疆天山2000-2017年植被水分利用效率(WUE)偏离 分析。

Fig. 2 Annual deviation analysis of vegetation water use efficiency (WUE) in Tianshan Mountains in Xinjiang during 2000 to 2017.
$\mathrm{C} \cdot \mathrm{mm}^{-1} \cdot \mathrm{m}^{-2}$, 和 $0.84 \mathrm{~g} \mathrm{C} \cdot \mathrm{mm}^{-1} \cdot \mathrm{m}^{-2}$, 低于多年平均 的 $22.52 \%$ 和 $24.32 \%$ 。

新疆天山2000-2017年近18年植被多年平均 WUE 呈明显的空间分异特征(图3), 垂直地带性的 影响显著, 海拔高度越高, WUE值越低, 变化范围 在0.000 1-1.08 $\mathrm{g} \mathrm{C} \cdot \mathrm{mm}^{-1} \cdot \mathrm{m}^{-2}$, 最高值分布在阿克 苏、渭干河流域及塔里木河干流区域, 该区域主要 是以耕种为主, 多为季节性农作物, 作物主要有小 麦、玉米和棉花, WUE值 $0.5 \mathrm{~g} \mathrm{C} \cdot \mathrm{mm}^{-1} \cdot \mathrm{m}^{-2}$ 以上, 最低 值分布在海拔高于 $2500 \mathrm{~m}$ 的区域, 主要以草地、林地 为主, $W U E$ 值在 $0.0001-0.08 \mathrm{~g} \mathrm{C} \cdot \mathrm{mm}^{-1} \cdot \mathrm{m}^{-2}$ 之间。

2000-2017年新疆天山植被WUE变化趋势在 -0.095-0.087 $\mathrm{g} \mathrm{C} \cdot \mathrm{mm}^{-1} \cdot \mathrm{m}^{-2}$ 之间(图4), 平均变化趋 势为 $-0.003 \mathrm{~g} \mathrm{C} \cdot \mathrm{mm}^{-1} \cdot \mathrm{m}^{-2}$ 。WUE呈增加趋势的范围 占全区 $16.68 \%$, 其中显著增加 $(p<0.01)$ 的区域占

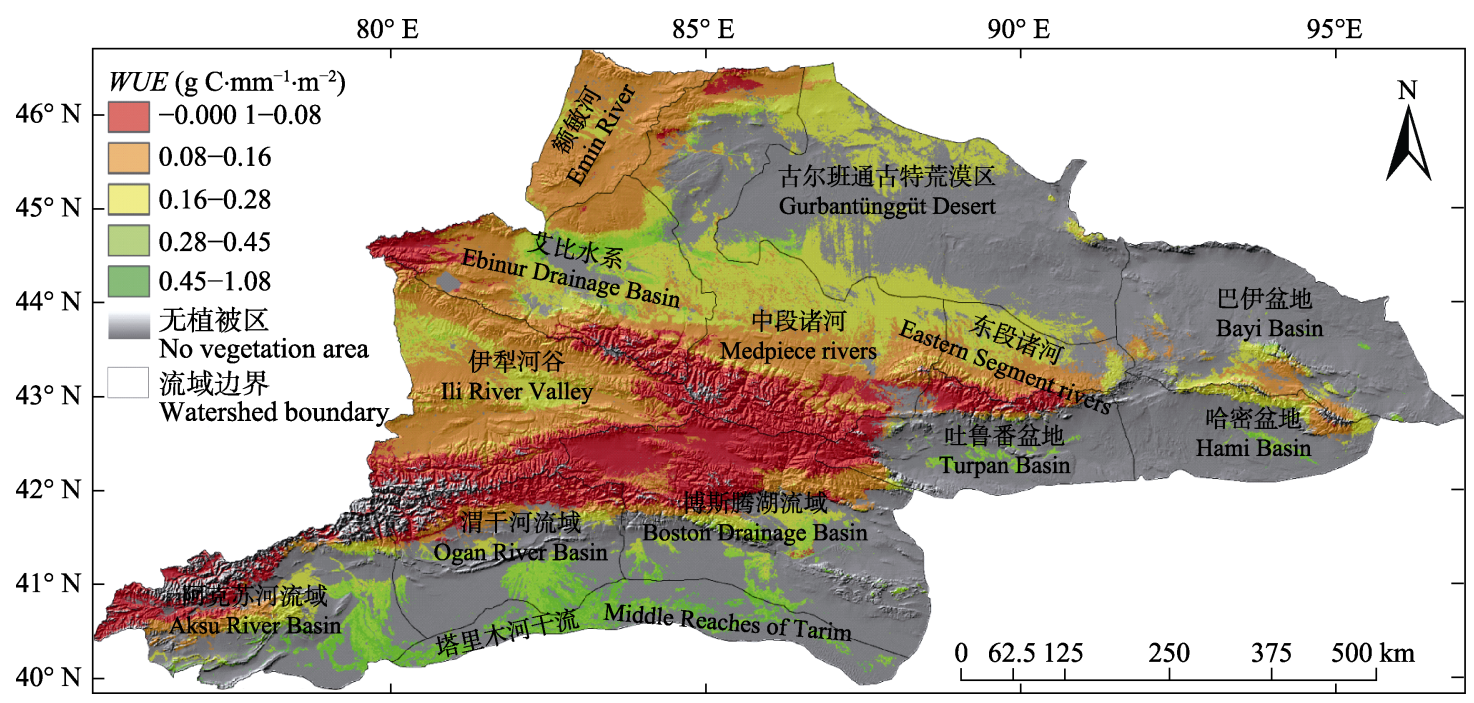

图3 新疆天山多年植被水分利用效率(WUE)空间分布。

Fig. 3 Spatial distributions of average vegetation water use efficiency (WUE) in Tianshan Mountains in Xinjiang.

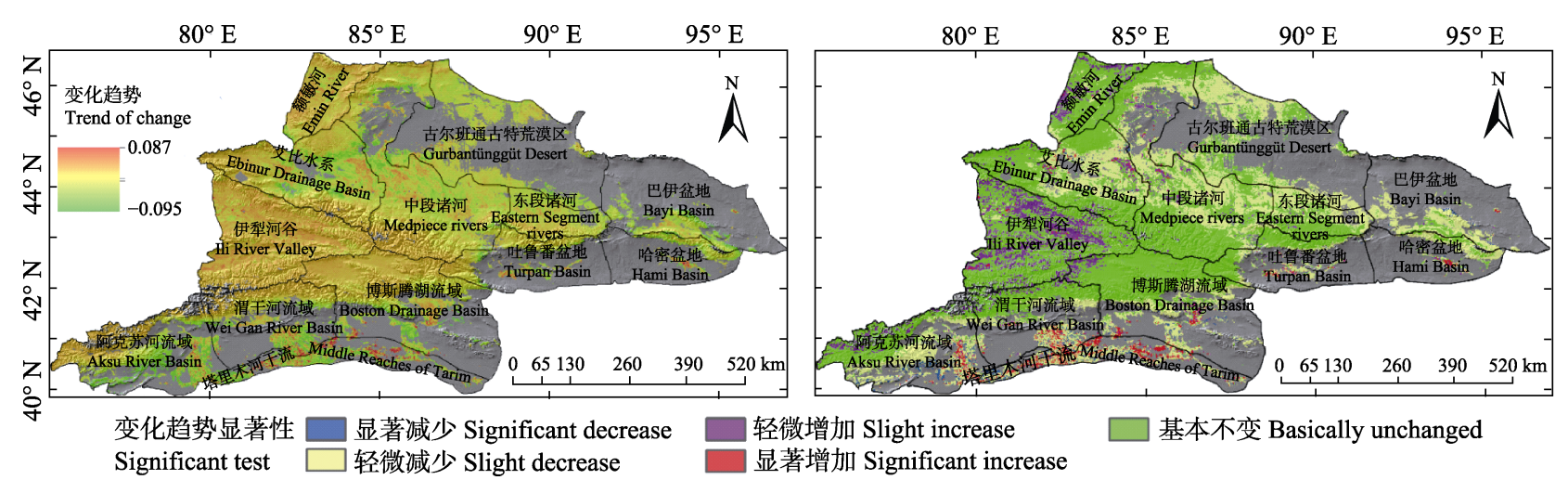

图4 新疆天山2000-2017年植被水分利用效率(WUE)变化趋势分布。

Fig. 4 The trend of vegetation water use efficiency (WUE) in Tianshan Mountains in Xinjiang during 2000 to 2017. 


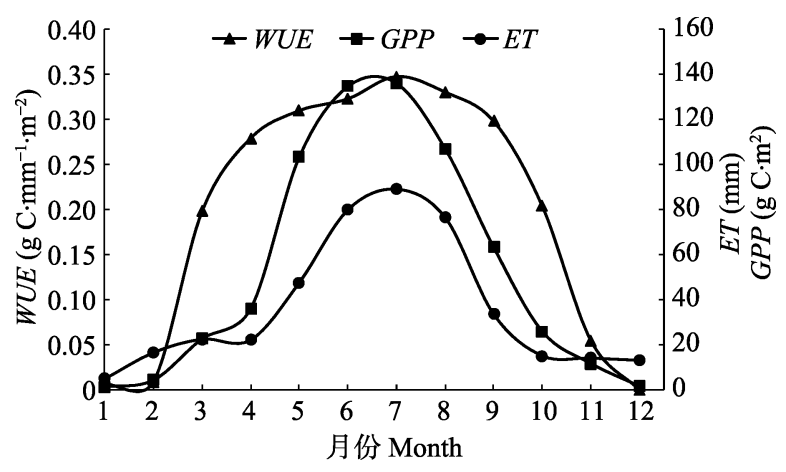

图5 新疆天山总初级生产力 $(G P P)$ 、蒸发散(ET)和植被水分 利用效率 $(W U E)$ 年内变化。

Fig. 5 Monthly variation of gross primary productivity $(G P P)$ 、 evaport transpration $(E T)$ and vegetation water use efficiency (WUE) in Tianshan Mountains in Xinjiang.

$2.56 \%$, 主要分布在阿克苏河、渭干河以及塔里木河 干流等区域; 轻微增加 $(p<0.05)$ 的区域占 $14.12 \%$, 主要出现在伊犁河谷和额敏河流域等区域。WUE呈 减少趋势的范围占全区的 $29.13 \%$, 其中显著减少 $(p<0.01)$ 的部分占 $1.25 \%$, 较分散地出现在阿克苏 河和塔里木河干流等区域; 轻微减少 $(p<0.05)$ 的 部分占 $27.88 \%$, 主要出现在天山北麓地区。WUE 基本不变的区域占 $54.18 \%$, 主要分布在海拔较高 的山区。

\section{2 天山地区植被WUE年内时空分布特征}

根据 MOD17A2H $8 \mathrm{~d}$ 合成 GPP 数据和 MOD16A2 $8 \mathrm{~d}$ 合成ET数据, 分析新疆天山植被 WUE的年内变化特征(图5), 新疆天山总初级生产 力年内变化呈现先增加后减少的单峰形趋势, 最大 值在7月份, 为 $136.21 \mathrm{~g} \mathrm{C} \cdot \mathrm{m}^{-2} ; E T$ 年内变化呈现双 峰形变化趋势, 最大值出现在 8 月份, 为 $89.21 \mathrm{~mm}$; 由于新疆天山及南北地区四季分异非常明显, 植被 $W U E$ 的年内变化呈现单峰形变化趋势, 夏季7月份 达到最高值, 为 $1.34 \mathrm{~g} \mathrm{C} \cdot \mathrm{mm}^{-1} \cdot \mathrm{m}^{-2}$, 冬季 12 月份达 到最低值。

由图6可以看到, 新疆天山不同季节植被WUE 的空间分布差异明显。春季(3-5月)气温回升阶段, 此阶段植被WUE值在 $0-0.68 \mathrm{~g} \mathrm{C} \cdot \mathrm{mm}^{-1} \cdot \mathrm{m}^{-2}$ 之间, 均 值为 $0.05 \mathrm{~g} \mathrm{C} \cdot \mathrm{mm}^{-1} \cdot \mathrm{m}^{-2}$, 高值出现在南部的阿克苏 河、渭干河及塔里木河干流等平原绿洲区域, 低值 分布在海拔2 $500 \mathrm{~m}$ 以上的高山区; 夏季(6-8月), 植 被WUE值范围为 $0-0.74 \mathrm{~g} \mathrm{C} \cdot \mathrm{mm}^{-1} \cdot \mathrm{m}^{-2}$, 均值为 0.07 $\mathrm{g} \mathrm{C} \cdot \mathrm{mm}^{-1} \cdot \mathrm{m}^{-2}$, WUE 高值分布在北部的古尔班通古 特沙漠区与周围的绿洲过渡带, 以及艾比湖周边, 低值主要分布在开孔河流域及北部海拔较高地区;
秋季(9-11月)开始降温、植被衰落阶段, WUE值范围 为 $0-0.52 \mathrm{~g} \mathrm{C} \cdot \mathrm{mm}^{-1} \cdot \mathrm{m}^{-2}$, 均值为 $0.03 \mathrm{~g} \mathrm{C} \cdot \mathrm{mm}^{-1} \cdot \mathrm{m}^{-2}$, 此阶段由于天山南部的降温比北部慢, 而且在阿克 苏、渭干河等流域还存在着未收完的棉花, 因此, WUE的高值还是出现在南部平原绿洲区, 低值主要 出现在巴音布鲁克草原及北部海拔高的地区; 冬季 (12月至次年2月)干冷阶段, WUE值范围为 $0-0.05 \mathrm{~g}$ $\mathrm{C} \cdot \mathrm{mm}^{-1} \cdot \mathrm{m}^{-2}$, 均值为 $0.0002 \mathrm{~g} \mathrm{C} \cdot \mathrm{mm}^{-1} \cdot \mathrm{m}^{-2}$, 此阶段 除天山南部的阿克苏地区和东部的吐鲁番地区外, 天山其他部分都被一层雪覆盖, 土壤基本冻结。整 体上新疆天山不同季节的植被WUE的空间分布情 况与太阳辐射、季节性气候、地形以及各种农作物 类型密切相关, 受气候条件和植被类型的影响, 新 疆天山植被 $W U E$ 整体上呈现夏季 $>$ 春季 $>$ 秋季 $>$ 冬季 的特征。

\section{3 不同土地利用类型的WUE变化特征}

土地利用类型变化是区域环境改变的直接结果, 它的变化在某种程度上影响区域环境的水文过程和 植被生产能力, 而 $E T$ 是地表水热平衡的重要构成部 分, $G P P$ 是植被的总初级生产力。因此, 探讨不同土 地利用类型的WUE特征具有重要的研究意义。本文 采用了2000、2005、2010和2015年4期土地类型数据, 分别代表2000-2004年、2005-2009年、2010-2013 年、2013-2017年4个时间段新疆天山土地利用情况, 分析不同土地类型的WUE变化特征(表1)。

从图7可知, 在不同时段内, 不同土地利用类型 的WUE值存在差异, 但各研究时段, 不同土地利用 类型的WUE值分布规律明显且相同: 水田 $>$ 其他林 地 $>$ 疏林地 $>$ 灌木林 $>$ 旱地 $>$ 低覆盖度草地 $>$ 有林地 $>$ 中覆盖度草地 $>$ 高覆盖度草地; 水田的WUE值明显 高于其他类型。可以看出, 所有土地类型的WUE随 时间变化均呈减少趋势, 水田与旱地类型WUE的持 续减少说明当地的人类活动存在一定的不合理性。

\section{4 新疆天山植被WUE与气候因子的关系分析}

气候变化显著影响陆地生态系统生产力以及 水分利用格局, 而WUE是衡量生态系统碳水耦合关 系的重要指标。因此, 研究陆地生态系统WUE对气 候变化的响应, 有助于深入了解生态系统的变化规 律, 模拟和预测生态系统碳水过程的发展状况(杜 晓铮等, 2018)。本文为了定量分析气候因子对新疆 天山植被WUE的影响，逐像元计算2000-2017年的 植被WUE与气温、降水之间的偏相关系数(图8)。 

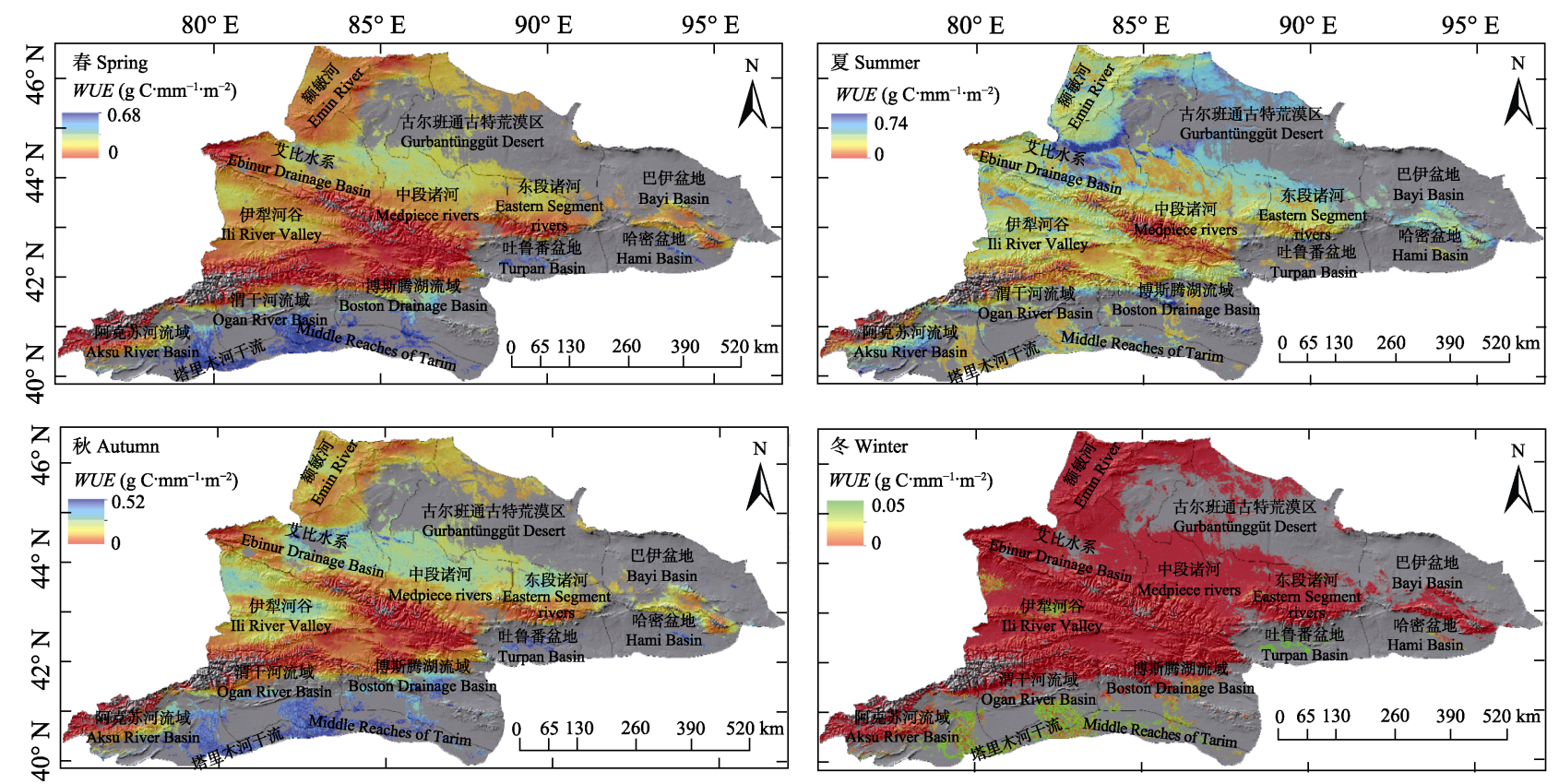

图6 新疆天山各季节植被水分利用效率(WUE)空间分布。

Fig. 6 Spatial distributions of vegetation water use efficiency (WUE) at four seasons in Tianshan Mountains in Xinjiang.

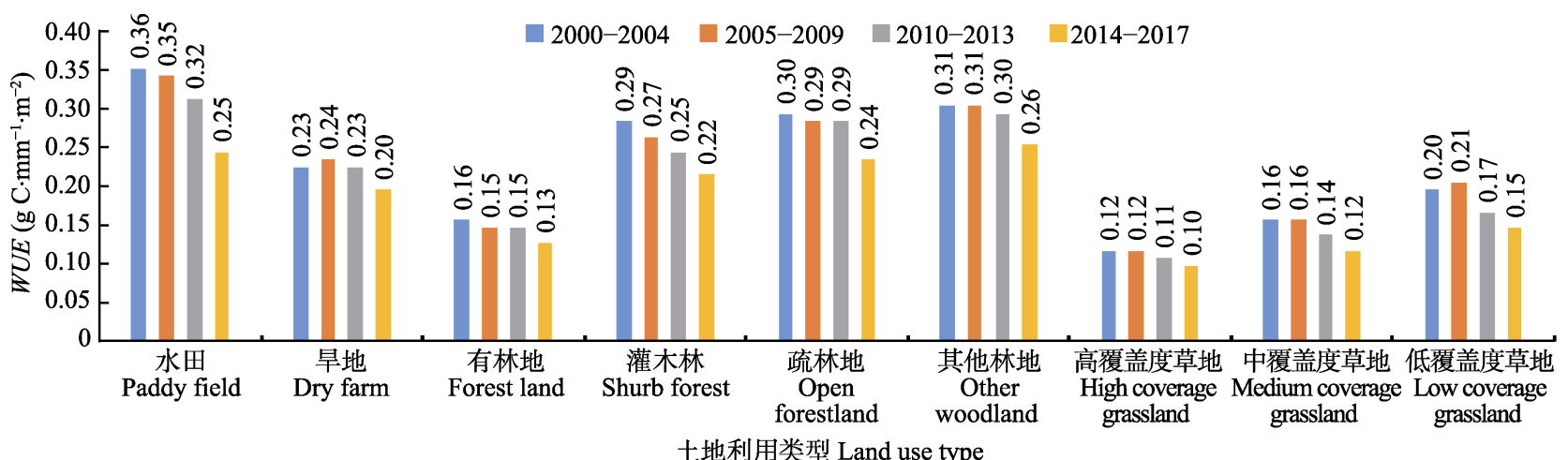

土地利用类型 Land use type

图7 新疆天山2000-2017年不同土地利用类型水分利用效率(WUE)随时间变化特征。

Fig. 7 Variation characteristics of water use efficiency (WUE) with time in different land use type in Tianshan Mountains in Xinjiang during 2000 to 2017 .
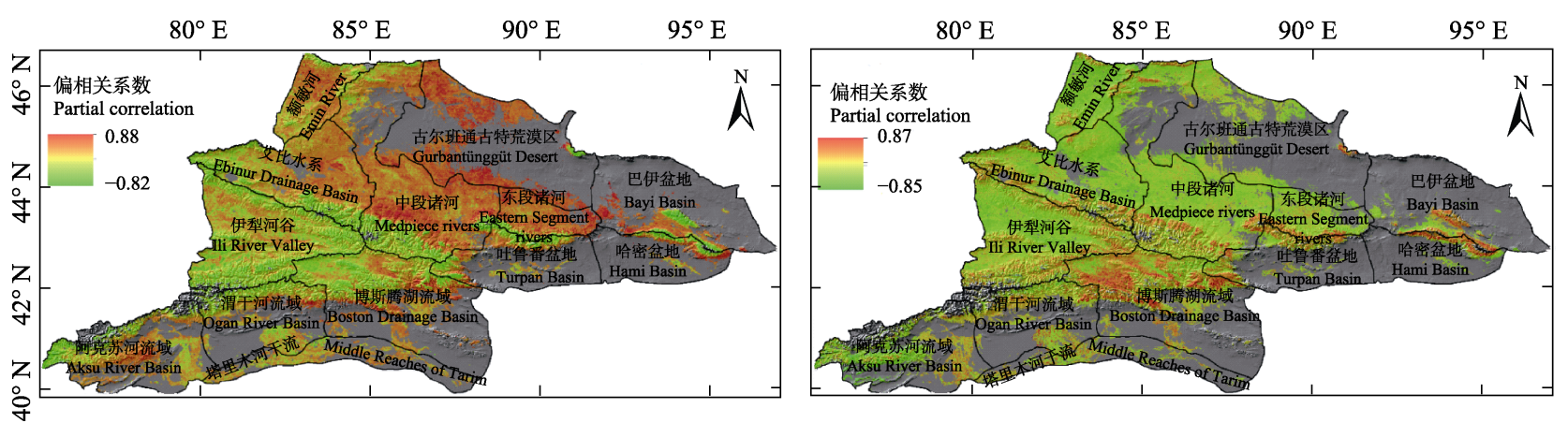

图8 新疆天山2000-2017年植被水分利用效率(WUE)与气温、降水的偏相关系数空间分布。

Fig. 8 Spatial distribution of partial correlations between vegetation water use efficiency (WUE) and temperature, precipitation in Tianshan Mountains in Xinjiang during 2000 to 2017.

从图中可以看出, 植被WUE对气候因子的响应表现 出较明显的空间差异性, 植被WUE与气温、降水的 偏相关系数分别介于为 $-0.82-0.88$ 和 $-0.85-0.87$ 之间,
统计结果表明, 植被WUE与气温、降水呈正相关关 系的面积分别占 $82.31 \%$ 和 $80.28 \%$, 主要分布在天山 两侧及伊犁河谷中部等海拔高度相对较低的地区;

www.plant-ecology.com 
呈负相关关系的区域主要分布在海拔较高的山区, 说明在整个研究区, 随着海拔的升高, 气温和降水 对植被WUE的影响逐渐变小, 其他因子的影响逐渐 变大。

由植被WUE与气温-降水的复相关系数空间分 布(图8)可知, WUE与气温-降水的复相关系数在 $0-0.88$ 之间, 平均值为 0.37 。整体而言, 植被WUE与 气温-降水的复相关性较强的区域主要集中在天山 北部地区古尔班通古特沙漠区周围的绿洲, 复相关 性较弱的区域主要分布在伊犁河谷以及开孔河流域 上游的巴音布鲁克草原地区。

为了更进一步得知气候因子对新疆天山植被 WUE的影响程度, 对之前计算出的偏相关性和复相 关性分别进行 $t$ 和 $F$ 检验, 然后参考众多学者所采用 的驱动分区原则(陈云浩等, 2001; 王强等, 2017; 艾 则孜提约麦尔·麦麦提等, 2018; 王芳等, 2018)对研 究区植被WUE变化进行驱动力分析, 其分类准则如 表3所示。
新疆天山植被WUE变化驱动分区结果如图9所 示, 结果表明, 气候变化对新疆天山植被WUE的影 响较明显, 通过统计得出, 2000-2017年研究区植被 WUE变化受到气温强驱动的区域占 $33.23 \%$, 各流域 在不同程度上受到气温的影响，其中对艾比湖、额 敏河、阿克苏河等流域的影响较明显; 降水强驱动 的区域面积占 $8.57 \%$, 其中对哈密盆地和巴伊盆地 的影响较显著; 气温降水共同强驱动的区域面积约 占 $5.63 \%$, 主要分布在开孔河流域; 气温降水弱驱 动的区域面积约占 $13.13 \%$, 主要分布在中段诸河与 东段诸河区域。整体上, 新疆天山南北两侧受气候 因子的影响较明显, 其中气温的影响力最大, 中间 海拔较高的山区受其他因子驱动。

\section{4 结论}

本文利用MODIS MOD16A2/ET与MOD17A2H/ GPP数据和研究区18年的气温、降水数据, 研究了 2000-2017年新疆天山植被WUE时空变化特征;

表1 水分利用效率(WUE)变化影响因子分区规则

Table 1 Analysis on driving factors for dynamic change of water use efficiency (WUE)

\begin{tabular}{|c|c|c|c|c|c|}
\hline \multicolumn{3}{|c|}{ WUE变化驱动因子 $W U E$ changes driving factors } & \multicolumn{3}{|c|}{ 分区准则 Rules } \\
\hline & & & $\mathrm{R} 1$ & $\mathrm{R} 2$ & $\mathrm{R} 3$ \\
\hline \multirow{4}{*}{$\begin{array}{l}\text { 气候因子 } \\
\text { Climate factors }\end{array}$} & 气温降水强驱动型 Changed by temperature and precipitation strongly & {$[\mathrm{T}+\mathrm{P}]^{+}$} & $|t|>t_{0.01}$ & $|t|>t_{0.01}$ & $F>F_{0.05}$ \\
\hline & 气温驱动型 Changed by temperature & $\mathrm{T}$ & $|t|>t_{0.01}$ & & $F>F_{0.05}$ \\
\hline & 降水驱动型 Changed by precipitation & $\mathrm{P}$ & & $|t|>t_{0.01}$ & $F>F_{0.05}$ \\
\hline & 气温降水弱驱动型 Changed by temperature and precipitation weakly & {$[\mathrm{T}+\mathrm{P}]^{-}$} & $|t| \leqslant t_{0.01}$ & $|t| \leqslant t_{0.01}$ & $F>F_{0.05}$ \\
\hline $\begin{array}{l}\text { 非气候因子 } \\
\text { Non-climate fact }\end{array}$ & 非气候因子驱动型 Changed by non-climate & $\mathrm{NC}$ & & & $F \leqslant F_{0.05}$ \\
\hline
\end{tabular}

$\mathrm{R} 1, W U E$ 与气温偏相关的 $t$ 显著性检验; R2,WUE与降水偏相关的 $t$ 显著性检验; R3,WUE与气温、降水复相关的 $F$ 显著性检验。

$\mathrm{R} 1, t$-Test significance of the partial correlations between $W U E$ and temperature; R2, $t$-Test significance of the partial correlations between $W U E$ and precipitation; R3, F-Test significance of the multiple correlations between WUE and temperature-precipitation.
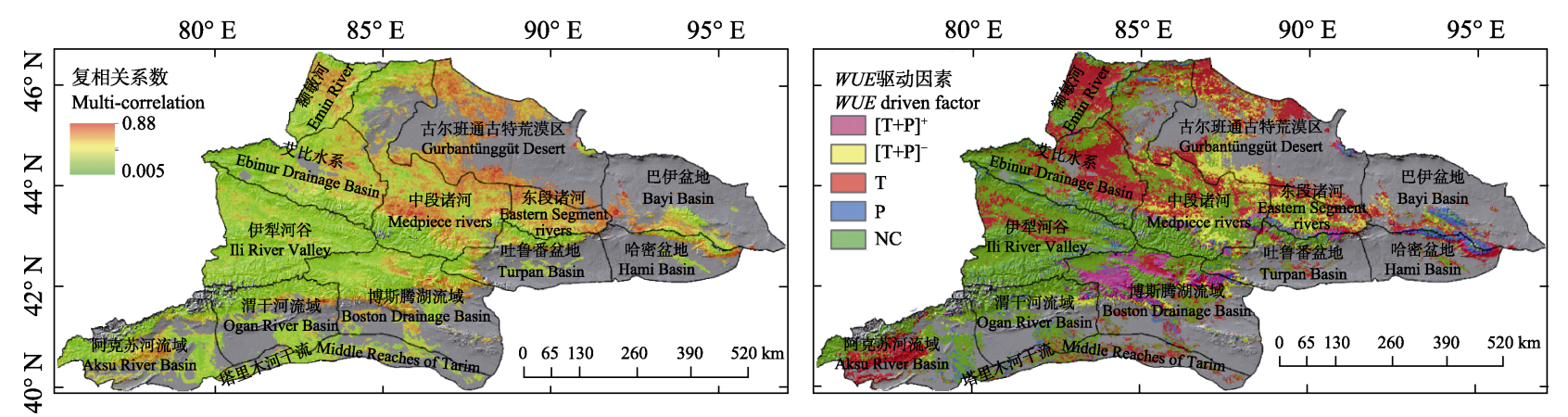

图9 新疆天山2000-2017年植被水分利用效率(WUE)与气温-降水的复相关分布和 $W U E$ 变化驱动力分区。 $[\mathrm{T}+\mathrm{P}]^{+}$, 气温、降水 强驱动; $\mathrm{T}$, 气温为主驱动; $\mathrm{P}$, 降水为主驱动; $[\mathrm{T}+\mathrm{P}]^{-}$, 气温降水弱驱动; $\mathrm{NC}$, 非气候驱动。

Fig. 9 Spatial distribution of multiple correlation between vegetation water use efficiency (WUE) and temperature-precipitation, and WUE change driven by different factors from 2000 to 2017 in Tianshan Mountains in Xinjiang. $[\mathrm{T}+\mathrm{P}]^{+}$, change driven by temperature and precipitation strongly; $\mathrm{T}$, change driven by temperature mainly; $\mathrm{P}$, change driven by precipitation mainly; $[\mathrm{T}+\mathrm{P}]^{-}$, change driven by temperature and precipitation weakly; $\mathrm{NC}$, change driven by non-climate. 
采用趋势分析法和相关性分析法, 探索了新疆天山 植被WUE与气温降水等气候因子的关系并最终得 到了驱动分区。主要结论如下:

(1) 2000-2017年新疆天山年际 $E T$ 与 $G P P$ 均呈增 长趋势, 但 $E T$ 的变化率大于 $G P P$, 导致年际植被 WUE的减少趋势。2000-2017年各年植被WUE偏离 多年平均水平的程度较大，其中2003、2005、2010、 2011 、2013年和最后3年的偏差偏离平均水平的程度 较大。2000-2017年植被多年平均WUE呈明显的空 间分异特征，变化范围为 $0-1.08 \mathrm{~g} \mathrm{C} \cdot \mathrm{mm}^{-1} \cdot \mathrm{m}^{-2}$ 之间， 整体空间分布表现出垂直地带性。多年空间变化趋 势为-0.094-0.066 $\mathrm{g} \mathrm{C} \cdot \mathrm{mm}^{-1} \cdot \mathrm{m}^{-2} \cdot \mathrm{a}^{-1}$ 之间, 平均变化 趋势为 $-0.003 \mathrm{~g} \mathrm{C} \cdot \mathrm{mm}^{-1} \cdot \mathrm{m}^{-2} \cdot \mathrm{a}^{-1}, 83.32 \%$ 的面积呈减 少趋势。

(2) 2000-2017年新疆天山年内植被WUE与 $G P P$ 呈周期性单峰形变化趋势, 最高值均出现在7月份, 但 $E T$ 呈双峰形变化趋势, 最高值出现在 8 月份; 新 疆天山各流域年内植被WUE呈较复杂的地域性, 这 可能与各流域气候、地形以及物候特征有关。不同 季节植被WUE的空间分布差异显著, 不同季节的植 被WUE大小关系为: 夏季 $>$ 春季 $>$ 秋季 $>$ 冬季。

(3) 新疆天山植被WUE变化受气候因子影响的 区域占 $60.74 \%$, 主要分布在天山南北以及伊犁河谷 海拔较低区域, 其中气温主驱动区域占 $33.23 \%$, 降 水主驱动区域约占 $8.57 \%$, 气温和降水共同强驱动 区域占约 $5.63 \%$, 气温降水共同弱驱动区域占 $13.13 \%$ 。

将研究区土地利用类型分布与驱动因子分析结 果(图8)相结合来看, 阿克苏河流域、渭干河流域以 及天山北坡的耕地(水田、旱地)区域基本上受非气 候因子的影响, 说明当地人类活对该区域的影响较 明显, 并且水田旱地等类型水分利用效率随时间持 续减少更进一步揭示了当地人类活动中存在一定的 不合理性。

\section{参考文献}

An YZ, Gao W, Gao ZQ, Shi RH (2016). Autumn vegetation change and response on climate change in northern China. Geomatics \& Spatial Information Technology, 39, 25-29, 35. [安佑志, 高炜, 高志强, 施润和 (2016). 中国北方 地区秋季植被变化及对气候变化的响应研究. 测绘与 空间地理信息, 39, 25-29, 35.]

Chen HB, Diao LJ (2005). Some extreme events of weather, climate and related phenomena in 2004. Climatic and Environmental Research, 10, 140-144. [陈洪滨, 刀丽军 (2005). 2004年的极端天气和气候事件及其他相关事件 的概要回顾. 气候与环境研究, 10, 140-144.]

Chen XG, Pan WJ, Zhang JY, Luo XL (2007). The extreme climate events in Guangzhou are increased due to the significant warming of the climate. Guangdong Meteorology, 29, 24-25. [陈新光, 潘蔚娟, 张江勇, 罗晓玲 (2007). 气候显著变暖使广州极端气候事件增多. 广东气象, 29 , 24-25.]

Chen YH, Li XB, Shi PJ (2001). Variation in NDVI driven by climate factors across China, 1983-1992. Acta Phytoecologica Sinica, 25, 716-720. [陈云浩, 李晓兵, 史培 军 (2001). 1983-1992年中国陆地NDVI变化的气候因子 驱动分析. 植物生态学报, 25, 716-720.]

Dilixiati M, Rusuli Y, Jiang H (2018). Temporal and spatial variation of vegetation phenology and its driving factor analysis in the Bosten Lake Drainage Basin from 2001 to 2014. Acta Ecologica Sinica, 38, 6921-6931. [玛地尼亚 提・地里夏提, 玉素甫江・如素力, 姜红 (2018). 20012014年博斯腾湖流域植被物候时空变化及其驱动因子. 生态学报, 38, 6921-6931.]

Du XZ, Zhao X, Wang HY, He B (2018). Responses of terrestrial ecosystem water use efficiency to climate change: A review. Acta Ecologica Sinica, 38, 8296-8305. [杜晓铮, 赵祥, 王吴宇, 何斌 (2018). 陆地生态系统水分利用效 率对气候变化的响应研究进展. 生态学报, 38, 8296-8305.]

Gang CC, Wang ZQ, Chen YZ, Yang Y, Li JL, Cheng JM, Qi JG, Odeh I (2016). Drought-induced dynamics of carbon and water use efficiency of global grasslands from 2000 to 2011. Ecological Indicators, 67, 788-797.

Heinsch FA, Reeves M, Votava P, Kang S, Milesi C, Zhao M, Glassy J, Jolly WM, Loehman R, Bowker CF, Kimball JS, Nemani RR, Running SW (2003). User's guide: GPP and NPP (MOD17A2/A3) Products NASA MODIS Land Algorithm, Version 2.0. The University of Montana, Missoula. 31-33.

Hu ZM, Yu GR, Wang QF, Zhao FH (2009). Ecosystem level water use efficiency: A review. Acta Ecologica Sinica, 29, 1498-1507. [胡中民, 于贵瑞, 王秋风, 赵风华 (2009). 生态系统水分利用效率研究进展. 生态学报, 29 , 1498-1507.]

Jassal RS, Black TA, Spittlehouse DL, Brümmer C, Nesic Z (2009). Evapotranspiration and water use efficiency in different-aged Pacific Northwest Douglas-fir stands. Agricultural and Forest Meteorology, 149, 1168-1178.

Klein T, Shpringer I, Fikler B, Elbaz G, Cohen S, Yakir D (2013). Relationships between stomatal regulation, wateruse, and water-use efficiency of two coexisting key Mediterranean tree species. Forest Ecology and Management, $302,34-42$. 
Li HD, Guan DX, Yuan FH, Wang AZ, Jin CJ, Wu JB, Li Z, Jing YL (2015). Water use efficiency and its influential factor over Horqin Meadow. Acta Ecologica Sinica, 35, 478-488. [李辉东, 关德新, 袁风辉, 王安志, 金昌杰, 吴家兵, 李峥, 井艳丽 (2015). 科尔沁草甸生态系统水 分利用效率及影响因素. 生态学报, 35, 478-488.]

Li MX, Yang YZ, Zhu QA, Chen H, Peng CH (2016). Evaluating water use efficiency patterns of Qinling Mountains under climate change. Acta Ecologica Sinica, 36, 936-945. [李明旭, 杨延征, 朱求安, 陈槐, 彭长辉 (2016). 气候 变化背景下秦岭地区陆地生态系统水分利用率变化趋 势. 生态学报, 36, 936-945.]

Li XJ, Zhang FP, Wang HW, Lei SJ, Gao Z (2017). Analysis of the spatio-temporal characteristics of water use efficiency of vegetation and its relationship with climate in the Heihe river basin. Journal of Desert Research, 37, 733-741. [李 肖娟, 张福平, 王虎威, 雷声剑, 高张 (2017). 黑河流 域植被水分利用效率时空变化特征及其与气候因子的 关系. 中国沙漠, 37, 733-741.]

Li YJ, Ding JL, Zhang JY, Wu PF (2019). Response of vegetation cover to drought in the northern slope of the Tianshan Mountains during 2001-2015 based on the land-use and land-cover change. Acta Ecologica Sinica, 39, 6206-6217. [李艳菊, 丁建丽, 张钧泳, 武鹏飞 (2019). 基于土地利 用/土地覆盖分析的2001-2015年天山北坡植被覆盖对 干旱的响应. 生态学报, 39, 6206-6217.]

Liu X, Qi C, Yan YL, Yuan GF (2017). Revised algorithm of ecosystem water use efficiency for semi-arid steppe in the Loess Plateau of China. Chinese Journal of Plant Ecology, 41，497-505. [刘晓, 戚超, 问艺兰, 袁国富 (2017). 不 同生态系统水分利用效率指标在黄土高原半干旱草地 应用的适宜性评价. 植物生态学报, 41, 497-505.]

Lu L, Li X, Huang CL, Frank V (2007). Analysis of the spatio-temporal characteristics of water use efficiency of vegetation in west China. Journal of Glaciology and Geocryology, 29, 777-784. [卢玲, 李新, 黄春林, Frank $\mathrm{V}$ (2007). 中国西部植被水分利用效率的时空特征分析. 冰川冻土, 29, 777-784.]

Lyapustin A, Wang Y, Xiong X, Meister G, Platnick S, Levy R, Franz B, Korkin S, Hilker T, Tucker J, Hall F, Sellers P, Wu A, Angal A (2014). Scientific impact of MODIS C5 calibration degradation and $\mathrm{C} 6+$ improvements. Atmospheric Measurement Techniques, 7, 4353-4365.

Maimaiti A, Rusuli Y, Jiang H, Abudukerimu B, He H (2018). Spatio-temporal variations and impacting factors of vegetation NPP in Bosten Lake Drainage Basin from 2000 to 2014. Pratacultural Science, 35, 1743-1753. [艾则孜提约 麦尔・麦麦提, 玉素甫江・如素力, 姜红, 拜合提尼沙・阿 不都克日木, 何辉 (2018). 2000-2014年博斯腾湖流域 NPP时空变化特征及影响因子分析. 草业科学, 35, 1743-1753.]

Qiu KB, Cheng JF (2015). Vegetation water use efficiency and its relationship with climate in Shaanxi Province. Research of Soil and Water Conservation, 22, 256-260. [仇宽彪, 成军锋 (2015). 陕西省植被水分利用效率及与气候因 素的关系. 水土保持研究, 22, 256-260.]

Running SW, Mu QZ, Zhao MS, Moreno A (2018). User's Guide MODIS Global Terrestrial Evapotranspiration (ET) Product (NASA MOD16A2/A3) NASA Earth Observing System MODIS Land Algorithm, Version 1.6. The University of Montana, Missoula. 20-33.

Running SW, Zhao MS (2015). User's Guide Daily GPP and NPP (MOD17A2/A3) Products NASA Earth Observing System MODIS Land Algorithm Version 3.0. Land Processes Distributed Active Archive Center, South Dakota. 9-14.

Wang F, Wang Z, Huang J, Yang SJ, He GJ, Zhang Y (2018). Spatio-temporal distribution characteristics of cropland water use efficiency and its relationship with climate in Anhui Province. Acta Ecologica Sinica, 38, 6268-6279. [王芳, 汪左, 黄静, 杨淑杰, 贺广均, 张运 (2018). 安 徽省农田水分利用效率时空特征及其与气候因子的关 系. 生态学报, 38, 6268-6279.]

Wang J, Jiang DK, Zhang YJ (2012). Analysis on spatial and temporal variation of extreme climate events in North China. Chinese Journal of Agrometeorology, 33, 166-173. [王冀, 蒋大凯, 张英娟 (2012). 华北地区极端气候事 件的时空变化规律分析. 中国农业气象, 33, 166-173.]

Wang LC, Zhu HJ, Lin AW, Zou L, Qin WM, Du QY (2017). Evaluation of the latest MODIS GPP products across multiple biomes using global eddy covariance flux data. Remote Sensing, 9, 418. DOI: 10.3390/rs9050418.

Wang Q, Zhang TB, Yi GH, Chen TT, Bie XJ, He YX (2017). Tempo-spatial variations and driving factors analysis of net primary productivity in the Hengduan mountain area from 2004 to 2014. Acta Ecologica Sinica, 37, 3084-3095. [王强, 张廷斌, 易桂花, 陈田田, 别小娟, 何奕萱 (2017). 横断山区2004-2014年植被NPP时空变化及其 驱动因子. 生态学报, 37, 3084-3095.]

Wang QW, Yu DP, Dai LM, Zhou L, Zhou WM, Qi G, Qi L, Ye YJ (2010). Research progress in water use efficiency of plants under global climate change. Chinese Journal of Applied Ecology, 21, 3255-3265. [王庆伟, 于大炮, 代力 民, 周莉, 周旺明, 齐光, 齐麟, 叶雨静 (2010). 全球气 候变化下植物水分利用效率研究进展. 应用生态学报, 21, 3255-3265.]

Wei HJ, Zhang YF, Dong XB, Lu NC, Wang XC (2016). Estimating the spatio-temporal characteristic of vegetation water use efficiency over Weihe River basin. Journal of Natural Resources, 31, 1275-1288. [位贺杰, 张艳芳, 董 孝斌, 鲁纳川, 王雪超 (2016). 渭河流域植被WUE遥感 估算及其时空特征. 自然资源学报, 31, 1275-1288.]

Wei WS, Hu RJ (1990). Precipitation and climate conditions of Tianshan Mountains. Arid Land Geography, 13, 29-36. 
[魏文寿, 胡汝瀷 (1990). 中国天山的降水与气候效应. 干旱区地理, 13, 29-36.]

Wu XQ, Wang RH, Li C, Jiang YL, Peng Q, Li Y (2016). Spatial-temporal characteristics of NPP and its response to climatic factors in Tianshan Mountains region. Ecology and Environmental Sciences, 25, 1848-1855. [吴晓全, 王 让会, 李成, 蒋烨林, 彭擎, 李炎 (2016). 天山植被 NPP时空特征及其对气候要素的响应. 生态环境学报, 25, 1848-1855.]

Wufu A, Rusuli Y, Kadeer R, Jiang H (2017). Spatio-temporal distribution and evolution trend of evapotranspiration in Xinjiang based on MOD16 data. Geographical Research, 36, 1245-1256. [阿迪来・乌甫, 玉素甫江・如素力, 热伊 莱・卡得尔, 姜红 (2017). 基于MODIS数据的新疆地表 蒸散量时空分布及变化趋势分析. 地理研究, 36, 1245-1256.]

Zhang YD, Pang R, Gu FX, Liu SR (2016). Temporal-spatial variations of WUE and its response to climate change in alpine area of southwestern China. Acta Ecologica Sinica, 36, 1515-1525. [张远东, 庞瑞, 顾峰雪, 刘世荣 (2016). 西南高山地区水分利用效率时空动态及其对气候变化 的响应. 生态学报, 36, 1515-1525.]

Zheng JY, Hao ZX, Fang XQ, Ge QS (2014). Changing characteristics of extreme climate events during past 2000 years in China. Progress in Geography, 33, 3-12. [郑景 云, 郝志新, 方修琦, 葛全胜 (2014). 中国过去2000年 极端气候事件变化的若干特征. 地理科学进展, 33, 3-12.]

Zhu HJ, Lin AW, Wang LC, Xia Y, Zou L (2016). Evaluation of MODIS gross primary production across multiple biomes in China using eddy covariance flux data. Remote Sensing, 8, 395. DOI: 10.3390/rs8050395.

责任编委: 陈世苹 责任编辑: 李 敏 实习编辑: 赵 航 